\title{
Organic matter sulfurization as a mechanism of enhanced burial of reduced carbon and sulfur across the Toarcian Oceanic Anoxic Event
}

\author{
Marroquin, S. M. ${ }^{1}$, Pritchard, J. A. ${ }^{1}$, Föllmi, K. B. ${ }^{2}$, \\ FANTASIA, A. ${ }^{3}$, RUEBSAM, W..$^{4}$, Trabucho \\ ALEXANDRE, J. P. ${ }^{5}$, GILl, B. C. ${ }^{1}$ \\ ${ }^{1}$ Virginia Tech, Blacksburg, VA 24060, USA \\ (*correspondence: selva@vt.edu) \\ ${ }^{2}$ University of Lausanne, Lausanne, 1015, Switzerland \\ ${ }^{3}$ Aarhus University, Aarhus C, Denmark \\ (alicia.fantasia@unil.ch) \\ ${ }^{4}$ University of Kiel, Ludewig-Meyn Str. 10, Kiel, 24118 , \\ Germany (wolfgang.ruebsam@ifg.uni-kiel.de) \\ ${ }^{5}$ Utrecht University, P.O. Box 80115,3508 TC Utrecht, The \\ Netherlands (J.Trabucho@uu.nl)
}

A fundamental debate exists as to what mechanism exerts the dominant control on the formation of organic-matter-rich mudstones: enhanced organic matter production or preservation. Organic matter sulfurization (OMS) is one process that directly enhances preservation by making organic matter less bioavailable. OMS operates most rapidly within euxinic water columns. Here, we investigated OMS as a potential pathway of enhanced preservation of organic matter across the Toarcian Oceanic Anoxic Event (TOAE, $\sim 183 \mathrm{Ma}$ ) of the Early Jurassic. We will present data from the event as recorded within three basins of the European epicontinental seaway. We find that the ratio of S:C in organic matter increases systematically with TOC, maxing out at $3 \% \mathrm{~S}: \mathrm{C}$; and pyrite sulfur dominates as the reduced sulfur flux across all sections. When compared to other Mesozoic OAEs, we see similar overall positive correlations between increased $\mathrm{S}: \mathrm{C}$ ratio of organic matter with TOC, but much less sulfurization across the Lower Jurassic as compared to Cretaceous OAE 2. Our results show that OMS is not a consistent feature across all OAEs and further work is needed to constrain the importance of OMS on reduced sulfur and carbon burial in deep time. 